\title{
Modified inertia from extended uncertainty principle(s) and its relation to MoND
}

\author{
Jaume Giné $^{1, a}$, Giuseppe Gaetano Luciano ${ }^{2,3, b}$ \\ ${ }^{1}$ Departament de Matemàtica, Universitat de Lleida, Av. Jaume II, 69, 25001 Lleida, Catalonia, Spain \\ ${ }^{2}$ Dipartimento di Fisica, Università di Salerno, Via Giovanni Paolo II, 132, 84084 Fisciano, SA, Italy \\ ${ }^{3}$ INFN, Sezione di Napoli, Gruppo collegato di Salerno, Naples, Italy
}

Received: 4 August 2020 / Accepted: 30 October 2020 / Published online: 9 November 2020

(C) The Author(s) 2020

\begin{abstract}
In this paper we show that Modified Inertia, i.e., the modification of inertia predicted by some alternative theories of gravity at cosmic scales, can be naturally derived within the framework of the extended uncertainty principle (EUP). Specifically, we consider two possible extensions of the Heisenberg uncertainty principle (HUP), corresponding to two different deformations of the fundamental commutator: the first one provides the natural generalization of the HUP to the (anti)-de Sitter spacetime and is endowed with only a quadratic correction in the uncertainty position. On the other hand, the second model contains both linear and quadratic extra terms. We prove that modified inertia is a direct consequence of the minimal acceleration experienced by any body due to the cosmic expansion. The obtained results are then discussed in connection with the empirical predictions of Modified Newtonian dynamics (MoND). The requirement of consistency between the two approaches allows us to fix the adjustable constant which marks the transition between the Newtonian and deep-MoND regimes.
\end{abstract}

\section{Introduction}

The Heisenberg Uncertainty Principle (HUP) plays a crucial rôle within the framework of quantum mechanics $(\mathrm{QM})$ and can be considered as the cornerstone of the theory which successfully describes systems at microscopic level and relatively short distances. On the other hand, general relativity (GR) - the best theory yet of the gravitational interaction avails of classical laws to explain the physics of macroscopic bodies and large distance scales. These two very different theories manage to share centre stage by keeping clear of each other. Indeed, while quantum mechanical effects are typically

\footnotetext{
a e-mail: gine@matematica.udl.cat

b e-mail: gluciano@sa.infn.it (corresponding author)
}

negligible when dealing with gravitational phenomena, gravity becomes inconsequential in the quantum realm. In spite of these tensions, it is expected that GR and QM domains have a non-vanishing intersection, and the formulation of a unified theory is by far the greatest challenge in theoretical physics nowadays.

Among the various attempts to incorporate gravity in the quantum world, the quantum field theory in curved space has yielded great development in recent years. In particular, this has been achieved through the prediction of the Hawking [1] and Unruh [2] effects, which emerge from the information loss associated to the appearance of event horizons - the black-hole horizon in the former case, the Rindler ( i.e., uniformly accelerated) horizon in the latter. These two effects are now widely accepted, but concerns about their detectability are still being expressed $[3,4]$. Likewise, another fertile arena to explore the interplay between quantum and gravity features is provided by the Generalized Uncertainty Principle (GUP), which accounts for the existence of a minimal length at Planck scale through a suitable modification of the HUP [5-11]. Duality in phase space have also suggested another extension of the uncertainty relation that should hold on a background with a maximal measurable length. This is known as Extended Uncertainty Principle (EUP) [12-14]. However, due to the lack of experimental guidance, physics at the border between GR and QM is largely heuristic and speculative. Therefore, most of the above conjectures should be either tested via in-the-lab analogues [15-17] or studied in connection with related phenomena.

In this vein, in Refs. $[18,19]$ the Unruh effect has been proposed to explain the origin of the inertial mass of bodies. In these works it is predicted that, when objects accelerate in one direction, a Rindler horizon forms on the opposite side, reducing the Unruh radiation by an asymmetric (Rindlerscale) Casimir effect. Hence, more pressure will hit the object coming from the front than from the rear, and this gives rise 
to a net force which opposes the acceleration, just like inertia. Because of its quantum origin, this model is named quantized inertia (QI). Nevertheless, the implementation of this idea is not given and a priori it seems difficult to find the inertial mass (a constant property of any body) for any value of the acceleration. In fact, only a modification of the inertia is obtained in these works. For the case of a particle of Planck mass, an approximation has been addressed in Ref. [20].

An inherent feature of QI is that, in low acceleration environments (for instance, at the edges of galaxies), the Unruh waves lengthen and are also damped by the cosmic horizon, this time equally in all directions (Hubble-scale Casimir effect) [18]. As a result, the effective mass $m_{I}$ of a body should be modified as

$m_{I}=m\left(1-\frac{2 c^{2}}{a \Theta}\right)$,

where $c$ is the speed of light, $a$ is the magnitude of the acceleration relative to the surrounding matter and $\Theta=2 R_{\mathrm{U}}$ is twice the cosmic radius $R_{\mathrm{U}}$. Clearly, for relatively large accelerations (for example equal or greater than Earth's gravity), the second term in the brackets becomes negligible and the standard inertia is recovered. On the other hand, the minimal value of the acceleration predicted by QI is $a=2 c^{2} / \Theta[18]$, which does not allow $m_{I}$ to become negative. Recently, a more refined derivation of Eq. (1) has been proposed in Refs. $[21,22]$ by assuming that the property of inertia is caused by gradients in the energy of the photons of Unruh radiation. In spite of some criticism (see Ref. [23]), QI has been claimed to explain galaxy rotation and cosmic acceleration without invoking dark matter and dark energy, respectively $[18,24,25]$.

Starting from the outlined picture, in this paper we provide a novel perspective on the phenomenon (1), showing that it arises from the accelerated expansion of the Universe. In this sense, it can be naturally derived within the framework of the Extended Uncertainty Principle in de Sitter spacetime, where the correction to the standard inertia is found to be related to the background cosmic acceleration and, thus, to the cosmological constant value. By analogy with QI, we shall refer to our model as Modified Inertia. For the sake of completeness, we also consider an alternative generalization of the uncertainty relation, corresponding to a different deformation of the HUP. Our results are then compared with the empirical interpolating functions used in Modified Newtonian Dynamics (MoND) [26,27]. By requiring consistency between the two approaches, we manage to fix the arbitrary MoND constant, which turns out to be equal to the cosmic acceleration.

The remainder of the work is structured as follows: in Sect. 2 we review the derivation of Eq. (1) presented in Ref. [22] with some improvements and conceptual discussions. The same analysis is extended to the framework of the EUP(s) in Sect. 3. Conclusions and future perspectives are summarized in Sect. 4. The work is completed with an Appendix containing details about the connection between a given background metric and the corresponding form of the EUP.

\section{Quantized inertia from HUP}

In Refs. [21,22] QI was derived by assuming that the inertia of accelerated bodies is caused by the gradient in the energy of Unruh radiation perceived as a result of their motion ${ }^{1}$ (we remark that vanishing acceleration is forbidden by QI model). In turn, this energy was estimated by means of the HUP

$\Delta x \Delta p \sim \frac{\hbar}{2} \Longrightarrow \Delta x \Delta E_{p} \sim \frac{\hbar c}{2}$,

where $E_{p}=p c$ is the energy of the photons from Unruh radiation.

With reference to Fig. 1, let us consider an accelerated body of mass $m$ moving rightwards along the $x$-axis and denote by $a$ the modulus of its acceleration. ${ }^{2}$ From ordinary considerations of relativistic kinematics, one can infer that a Rindler horizon will appear in the opposite direction to the acceleration, at a distance $d=c^{2} / a$ far away from the body. On the other side, a specular rôle is played by the cosmic horizon at a far greater distance $\Theta / 2 \gg d$.

Now, it is well known that the quantum vacuum is filled up with virtual particle-antiparticle pairs randomly popping into existence and disappearing again. Clearly, if one of these fluctuations appears behind the Rindler horizon on the left side, it will never be able to reach the accelerating body. As a consequence, all the quantum fluctuations coming from the strip between the equivalent Rindler horizon (dashed line) and the cosmic horizon on the right side are not balanced by photons in the opposite region, giving rise to an asymmetric Casimir effect (see Fig. 1).

We wonder what is the temperature of this unbalanced radiation. To answer this question, let us observe that fluctuations of Unruh radiation, which come from a distance of the order of Rindler horizon, satisfy

$$
\Delta E_{\mathrm{U}} \sim \frac{\hbar a}{2 \pi c}=K_{B} T_{\mathrm{U}}
$$

\footnotetext{
1 The Unruh effect is the prediction that a uniformly accelerated observer sees its surroundings as a thermal bath of particles, even when moving in the vacuum of inertial observers [2]. Remarkably, deviations of Unruh effect from pure thermality have been obtained in various contexts with non-trivial results [28-32].

${ }^{2}$ Here we shall treat the case of motion along one axis. The same considerations hold true in more dimensions.
} 


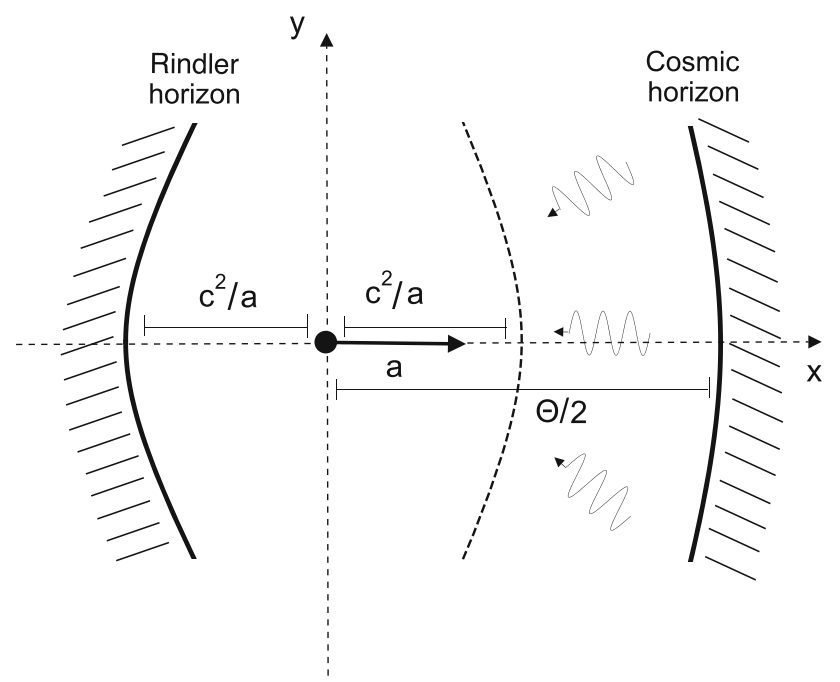

Fig. 1 Schematic representation of quantized inertia: a body (black dot) moving rightwards with acceleration $a$ perceives the cosmic horizon far away to its right (at a distance $\Theta / 2$ ) and a closer Rindler horizon to its left (at a distance $c^{2} / a$ ). Clearly, all the fluctuations originating within a distance $c^{2} / a$ on the right side are compensated by the symmetric action of fluctuations in the opposite region. By contrast, photons coming from the strip between the dashed line and the cosmic horizon on the right do not experience any balance. This horizon-scale Casimir effect produces a net force which opposes the acceleration, just like inertia

where $K_{B}$ is the Boltzmann constant and $T_{\mathrm{U}}$ is the wellknown Unruh temperature [2]

$T_{\mathrm{U}}=\frac{\hbar a}{2 \pi c K_{B}}$.

This relation can be easily obtained by using the HUP (2) and setting $\Delta x \sim \pi c^{2} / a$, where we are assuming a form of half circumference for the Rindler horizon as in Ref. [33]. Furthermore, since a quantum fluctuation is the temporary appearance of a particle-antiparticle pair, we can write $\Delta E_{\mathrm{U}} \sim 2 E_{\mathrm{U}}$, with $E_{\mathrm{U}}$ being the energy of each particle of the pair. Equation (3) then gives

$E_{\mathrm{U}} \sim \frac{K_{B} T_{\mathrm{U}}}{2}$.

The Unruh temperature is the consequence of the Unruh effect due to the quantum fluctuations near the horizon. However, this is a negligible phenomenon in the present analysis, since photons of the unbalanced radiation originate at distances greater than $\pi c^{2} / a$, as discussed above. Clearly, for such particles we have

$\Delta x \gtrsim \frac{\pi c^{2}}{a} \Longrightarrow T \lesssim T_{\mathrm{U}}$,

which states that the temperature $T$ of the unbalanced radiation is at most given by the Unruh temperature $T_{\mathrm{U}}$. Another different feature between the Unruh and unbalanced radiations is that, while the former is a very tiny effect which can only be detected by an observer comoving with the accelerating body, any inertial observer and everywhere in the space can observe the unbalance between photons coming from the front and the back of the body (the only requirement is that the body has a non-vanishing acceleration relative to its surroundings). Moreover, these photons are expected to produce macroscopic effects on the body, in the same way as vacuum fluctuations manifest themselves through an observable attractive force in the conventional Casimir effect.

Now, the idea proposed by McCulloch $[18,19]$ is that the asymmetric damping of quantum waves described above would be responsible for QI. Indeed, the unbalanced radiation originated by the appearance of Rindler and cosmic horizons exerts a non-vanishing pressure on the body, giving rise to a net force that pushes it back against its acceleration (see Fig. 1). In turn, such an effect can be rephrased in terms of a modification of the inertial mass of the body, which can be quantified by using the HUP and noticing that the energy uncertainty of fluctuations which impact on the body is

$\Delta E_{p} \sim \frac{\hbar c}{2}\left(\frac{1}{\Delta x_{2}}-\frac{1}{\Delta x_{1}}\right)$,

where $\Delta x_{1}$ and $\Delta x_{2}$ are the position uncertainties related to the cosmic and Rindler horizons, respectively. Based on the above considerations, we set $\Delta x_{1}$ equal to the half circumference of radius $R_{\mathrm{U}}$, i.e., $\Delta x_{1} \sim \pi R_{\mathrm{U}}=\pi \Theta / 2$, while $\Delta x_{2} \sim \pi d=\pi c^{2} / a$ as before. ${ }^{3}$ A straightforward substitution into Eq. (7) leads to

$\Delta E_{p} \sim \frac{\hbar a}{2 \pi c}\left(1-\frac{2 c^{2}}{a \Theta}\right)=K_{B} T_{\mathrm{U}}\left(1-\frac{2 c^{2}}{a \Theta}\right)$,

which can be expressed in terms of the energy $E_{p}=\Delta E_{p} / 2$ of each particle of the pairs belonging to the unbalanced radiation as

$E_{p} \sim E_{\mathrm{U}}\left(1-\frac{2 c^{2}}{a \Theta}\right)$

Now, let $E_{m} \sim m c^{2}$ be approximately the energy of the accelerating body. Here we adopt a corpuscular-like picture for the electromagnetic force [34] akin to the description of gravity proposed in Refs. [35,36]. Thus, we consider the body as a Bose-Einstein condensate of $N$ photons, which can be interpreted as the resonant parts that interact with the external radiation. Following Ref. [22], we then express $N$ as either the number of photons of Unruh radiation equivalent to the energy $E_{m}$ (since the standard mass-energy of the body is mostly determined by fluctuations coming from the Rindler horizon when QI effects are negligbile) or the number of photons of the unbalanced radiation equivalent to the reduced energy $E_{m}^{\prime} \sim m_{I} c^{2}$ (given that the modified inertia $m_{I}<m$

\footnotetext{
${ }^{3}$ In 3 dimensions $\Delta x_{1}$ becomes the half circumference of the Hubblesphere of radius $R_{\mathrm{U}}$. A similar generalization holds true for $\Delta x_{2}$.
} 
is due to the fluctuations of the unbalanced radiation in the QI regime). Mathematically speaking, we have $N=E_{m} / E_{\mathrm{U}}=$ $E_{m}^{\prime} / E_{p}$.

The above reasoning allows us to get the desired result. Indeed, by multiplying both sides of Eq. (9) by $N / c^{2}$, it follows that

$m_{I} \sim m\left(1-\frac{2 c^{2}}{a \Theta}\right)$

which is the same expression as Eq. (1). Note that, for $a \rightarrow 2 c^{2} / \Theta$, the modified inertia $m_{I}$ approaches to zero. By referring to the picture described above, this amounts to the physical setting where the Rindler and cosmic horizons emerge approximately at the same distance from the accelerating body. In this case the Unruh wavelengths will be damped in a symmetric way from both the front and the rear of the body, thus leading to an isotropic radiation pressure which cancels the effects of modified inertia.

Now, it is worth noting that Eq. (10) is consistent with the prediction of Modified Newtonian Dynamics (MoND) [26,27], which has been empirically derived from galaxy rotation data. Indeed, according to the MoND model with the simple interpolating function expanded to the leading order, Newton's second law of dynamics should be modified as follows

$$
\begin{aligned}
F_{0} & =m\left(1+\frac{a_{0}}{a}\right)^{-1} a \\
& \approx m\left[1-\frac{a_{0}}{a}+\mathscr{O}\left(\frac{a_{0}}{a}\right)^{2}\right] a,
\end{aligned}
$$

which can be interpreted in terms of a modification of the inertial mass as

$m_{i}=m\left[1-\frac{a_{0}}{a}+\mathscr{O}\left(\frac{a_{0}}{a}\right)^{2}\right]$.

Clearly, the agreement between Eqs. (10) and (12) is obtained, provided that the adjustable constant $a_{0}$ which marks the transition between the Newtonian and deep-MoND regimes is set equal to $2 c^{2} / \Theta$. Hence, the advantage of Modified Inertia is that it predicts corrections to the inertial mass without needing any arbitrary constant.

We also emphasize that the interpolating function is not uniquely fixed by MoND hypothesis, even though it can be partially constrained on empirical basis [37,38]. Apart from the model (11), another setting commonly found in literature is the so-called standard interpolating function

$$
\begin{aligned}
F_{0} & =m\left[1+\left(\frac{a_{0}}{a}\right)^{2}\right]^{-1 / 2} a \\
& \approx m\left[1-\frac{1}{2}\left(\frac{a_{0}}{a}\right)^{2}+\mathscr{O}\left(\frac{a_{0}}{a}\right)^{4}\right] a,
\end{aligned}
$$

which entails the modified inertia

$m_{i}=m\left[1-\frac{1}{2}\left(\frac{a_{0}}{a}\right)^{2}+\mathscr{O}\left(\frac{a_{0}}{a}\right)^{4}\right]$.

This differs from the expression in Eq. (12) as for the absence of the linear correction in $a_{0} / a$.

In the next section we show how Eqs. (12) and (14) can be straightforwardly derived from proper deformations of the HUP existing in literature.

\section{Quantized inertia from $\operatorname{EUP}(s)$}

Since a couple of decades, experimental data from supernovae, galaxy clusters and baryon acoustic oscillations have confirmed that our Universe is currently expanding at an accelerated rate. According to the models of inflation and observational evidences, there is quite general agreement that de Sitter spacetime is the most fitting candidate to describe our Universe in its early expanding phase and its far future.

Based on the above picture, we show that the modification in Eq. (14) can be naturally explained in the context of quantum mechanics in de Sitter spacetime, where it arises from the emergence of a characteristic length scale of the order of de Sitter radius. As a further model of modified inertia, we also comment on the possibility to trace Eq. (12) back to some suitable generalization of the uncertainty relation.

\subsection{Case I}

It has been argued that in the (anti)-de Sitter background, the HUP in Eq. (2) should be modified by introducing a correction proportional to the cosmological constant $|\Lambda| \sim 1 / l_{H}^{2}$ [12], i.e.,

$\Delta x \Delta p \sim \frac{\hbar}{2}\left[1+\frac{\gamma}{2}\left(\frac{\Delta x}{l_{H}}\right)^{2}\right]$.

Here $l_{H}$ is the (anti)-de Sitter radius and $\gamma$ the (dimensionless) deformation parameter, which is usually taken of order unity so that the extra term only becomes relevant at cosmological scales. In the following, we assume $\gamma<0(\gamma>0)$ for de Sitter (anti-de Sitter) spacetime. ${ }^{4}$ Clearly, for $\gamma=0$ and/or $\Delta x / l_{H} \ll 1$, the Heisenberg uncertainty relation (2) is recovered.

The uncertainty relation (15) is usually referred to as Extended Uncertainty Principle (EUP) [13,14] by analogy with the Generalized Uncertainty Principle (GUP) [5-11],

\footnotetext{
${ }^{4}$ Note that for $\gamma>0$ (anti-de Sitter spacetime), Eq. (15) implies the existence of a minimal momentum $p_{\min } \sim \frac{\hbar \sqrt{\gamma}}{l_{H}}$. On the other hand, for $\gamma<0$ (de Sitter spacetime), there is no constraint on the momentum, but it emerges a maximum length scale, which is given by the radius $l_{H}$ of the cosmological horizon.
} 
which is expected to hold at Planck (rather than cosmological) scales [39-48]. Phenomenological implications of the EUP have been considered in a variety of contexts, ranging from black hole physics $[13,49-52]$, to the thermodynamics of the FRW universe [49], the Unruh effect [52] and the Duffin-Kemmer-Petiau oscillator [53]. From a more theoretical perspective, the EUP has been investigated in Ref. [54], where it has been derived on the basis of first principles.

In order to show how the EUP (15) affects the inertia of an accelerating body, let us retrace the same steps as in Sect. 2. For this purpose, by using $E_{p}=p c$ for the photons of the unbalanced radiation, we get

$\Delta x \Delta E_{p} \sim \frac{\hbar c}{2}\left[1+\frac{\gamma}{2}\left(\frac{\Delta x}{l_{H}}\right)^{2}\right]$.

As discussed above, if the body moves with acceleration $a$, then it will perceive a Rindler horizon at a distance $d=$ $c^{2} / a<l_{H}$. The uncertainty of the crossing point of this horizon determines the uncertainty in the position of photons, which is still given by $\Delta x \sim \pi c^{2} / a$. Notably, this is the only scale we have to consider as a physical input in the present analysis.

From Eq. (16), the energy uncertainty of fluctuations of the environment radiation takes the form

$\Delta E_{p} \sim K_{B} T_{\mathrm{U}}\left[1+\frac{\gamma}{2}\left(\frac{\pi c^{2}}{l_{H} a}\right)^{2}\right]$,

which can be recast in terms of the energy $E_{p}$ of the single particle of each virtual pair as

$E_{p} \sim E_{\mathrm{U}}\left[1+\frac{\gamma}{2}\left(\frac{\pi c^{2}}{l_{H} a}\right)^{2}\right]$.

The above equation allows us to estimate the EUP-induced modification of the inertial mass of the body. Indeed, by introducing the number $N$ of resonant parts of the body and following the same considerations leading to Eq. (10), we obtain

$m_{I} \sim m\left[1+\frac{\gamma}{2}\left(\frac{\pi c^{2}}{l_{H} a}\right)^{2}\right]$.

At this stage, let us introduce the background cosmic acceleration $a_{H}=c^{2} / l_{H}$. Then, Eq. (19) takes the form

$m_{I} \sim m\left[1+\frac{\gamma \pi^{2}}{2}\left(\frac{a_{H}}{a}\right)^{2}\right]$.

For $\gamma=0$ and/or $a \gg a_{H}$, the standard inertia is recovered, consistently with the fact that the EUP in Eq. (15) reduces to the usual HUP (2) in these limits.

It is now easy to see that the Modified Inertia (20) has the same behavior as MoND prediction with the standard interpolating function, Eq. (14), provided that the condition

$\gamma=-\frac{1}{\pi^{2}}$ is satisfied. The negative value of the deformation parameter implies that MoND complies with the formulation of the EUP in de Sitter (rather than anti-de Sitter) spacetime, where a positive value of the cosmological constant yields an accelerated Universe expansion. Furthermore, the requirement of consistency between Eqs. (14) and (20) allows us to fix the MoND arbitrary constant $a_{0}$ equal to the background cosmic acceleration $a_{H}$. Therefore, in our model, Modified Inertia at cosmological scales is ascribed to the accelerating expansion of the Universe. This explains why such an effect is actually negligible in size for local (i.e., large acceleration environments) phenomena, for which $a \gg a_{H}$.

Let us now observe that Eq. (19) can be equivalently expressed as

$m_{I} \sim m\left(1-\frac{1}{2} \frac{c^{4} \Lambda}{a^{2}}\right)$,

which connects the mass correction to the value of the cosmological constant. Clearly, by use of this relation, one may constrain Modified Inertia by exploiting the current bound on the cosmological constant, that is $\Lambda \sim 10^{-52} \mathrm{~m}^{-2}$. For instance, for typical terrestrial acceleration $a \sim 10 \mathrm{~m} / \mathrm{s}^{2}$, we have

$\left|\frac{m_{I}-m}{m}\right| \sim \mathscr{O}\left(10^{-21}\right)$,

that is below the current experimental sensitivity, as expected. However, in environments with much smaller values of the acceleration, (for example at the edges of galaxies or in dwarf galaxies), this bound can be largely improved. For instance, for $a \sim 10^{-9} \mathrm{~m} / \mathrm{s}^{2}$, it follows that

$\left|\frac{m_{I}-m}{m}\right| \sim \mathscr{O}\left(10^{-1}\right)$,

which in principle may be tested through future highprecision measurements.

Now, an alternative way to derive Eq. (20) can be obtained by directly modelling the interaction between the unbalanced radiation and the test-body. In this regard, let us consider a quantum fluctiation of the unbalanced radiation. From Eq. (16), the energy of each photon of the pair can be estimated as

$E_{p} \sim \frac{\hbar c}{4 \Delta x}\left[1+\frac{\gamma}{2}\left(\frac{\Delta x}{l_{H}}\right)^{2}\right]$.

The above relation provides us with the value of the energy the photons transmit when impinging on the accelerating body. If the number of transmitted photons is $N$, then the total absorbed energy is

$E_{T} \sim \frac{N \hbar c}{4 \Delta x}\left[1+\frac{\gamma}{2}\left(\frac{\Delta x}{l_{H}}\right)^{2}\right]$. 
This energy yields a variation of the momentum of the body. In particular, the total transferred momentum is $p_{T}=E_{T} / c$. This transfer of momentum occurs via the exertion of a force

$m a=F=-\frac{\partial E_{T}}{\partial(\Delta x)}$.

By use of Eq. (26), this becomes

$m a \sim F_{0}\left[1-\frac{\gamma}{2}\left(\frac{\Delta x}{l_{H}}\right)^{2}\right]$,

where

$F_{0}=\frac{N \hbar c}{4(\Delta x)^{2}}$

is the radiation force acting on the body when EUP effects are neglected (i.e., for $\gamma=0$ ). Note that, in the present derivation, the arbitrary number $N$ of photons can be fixed by approximating the unbalanced radiation to a black-body radiation at temperature $T \lesssim T_{\mathrm{U}}$ (see Eq. (6)), and equating $F_{0}$ to the force exerted by such a radiation.

Equation (27) can be further manipulated by setting $\Delta x \sim$ $\pi c^{2} / a$ and $l_{H}=c^{2} / a_{H}$, as above. To the lowest order, we then obtain

$$
\begin{aligned}
m a & \sim F_{0}\left[1-\frac{\gamma \pi^{2}}{2}\left(\frac{a_{H}}{a}\right)^{2}\right] \\
& \Longrightarrow F_{0} \sim m\left[1+\frac{\gamma \pi^{2}}{2}\left(\frac{a_{H}}{a}\right)^{2}\right] a .
\end{aligned}
$$

As remarked in Eq. (14), the above correction can be reinterpreted in terms of a modification induced on the inertia of body as

$m_{I}=m\left[1+\frac{\gamma \pi^{2}}{2}\left(\frac{a_{H}}{a}\right)^{2}\right]$,

consistently with Eq. (20).

\subsection{Case II}

The extension of the HUP in Eq. (15) is not unique in literature. For instance, we can consider a modified uncertainty relation containing both linear and quadratic terms in $\Delta x / l_{H}$ (see Eq. (A.8) in the Appendix)

$\Delta x \Delta p \sim \frac{\hbar}{2}\left[1-\frac{\alpha}{2} \frac{\Delta x}{l_{H}}+\frac{3 \alpha^{2}}{2}\left(\frac{\Delta x}{l_{H}}\right)^{2}\right]$,

where $\alpha>0$ is the deformation parameter defined as in Eq. (A.5). We remark that a similar EUP has been analyzed in Ref. [54].

Following the same reasoning as in the previous analysis, we apply Eq. (33) to the photons of the unbalanced radiation, yielding

$$
\begin{aligned}
& \Delta x \Delta E_{p} \sim \frac{\hbar c}{2}\left[1-\frac{\alpha}{2} \frac{\Delta x}{l_{H}}+\frac{3 \alpha^{2}}{2}\left(\frac{\Delta x}{l_{H}}\right)^{2}\right] \\
& \Longrightarrow \Delta E_{p} \sim K_{B} T_{\mathrm{U}}\left[1-\frac{\alpha \pi}{2} \frac{a_{H}}{a}+\frac{3 \alpha^{2} \pi^{2}}{2}\left(\frac{a_{H}}{a}\right)^{2}\right] .
\end{aligned}
$$

This implies

$E_{p} \sim E_{\mathrm{U}}\left[1-\frac{\alpha \pi}{2} \frac{a_{H}}{a}+\frac{3 \alpha^{2} \pi^{2}}{2}\left(\frac{a_{H}}{a}\right)^{2}\right]$.

Once again, by expressing the energy of the environment radiation in terms of the the number of photons equivalent to the energy $E_{m}$ of the test-body, we obtain

$m_{I} \sim m\left[1-\frac{\alpha \pi}{2} \frac{a_{H}}{a}+\frac{3 \alpha^{2} \pi^{2}}{2}\left(\frac{a_{H}}{a}\right)^{2}\right]$.

This has the same behavior as MoND prediction (12), with the adjustable MoND parameter $a_{0}$ being given by the cosmic acceleration $a_{H}$, as above (the exact value of the coefficient $\alpha$ can be determined by equating the leading-order corrections in the two formulas, which gives $\alpha=2 / \pi$ ). Clearly, we would arrive at the same result by following the approach based on the direct computation of the radiation force.

A comment is in order here: as discussed in the Appendix, the EUP (33) naturally arises from the first terms in the expansion of a metric which has no evident physical meaning. Hence, the above considerations and the result (36) should only be considered as a mathematical framework to derive Eq. (1) from a deformed commutator. Beyond formal aspects, however, we expect that further details about the actual dependence of Modified Inertia on the acceleration either linear or quadratic in $1 / a$ - can be provided by future observational data, which will then reinforce the study of the model (20) or (36).

\section{Summary and conclusions}

The feature of inertial mass of bodies has never been well understood theoretically, but just assumed as empirical Newton's first law. Based on McCulloch's idea, which traces the origin of QI back to the combination of relativity (the appearance of the horizons) and quantum mechanics (the Casimir effect), here we have shown that a modification of the inertial mass can be naturally explained within the framework of the Extended Uncertainty Principle. Specifically, we have considered two different deformations of the standard Heisenberg relation. The first one only contains a quadratic correction in the uncertainty position and is the generalization which emerges in (anti)-de Sitter spacetime. In this context, 
we have found that Modified Inertia is related to the accelerating expansion of the Universe. Moreover, the obtained formula for the modified inertial mass is in agreement with the standard interpolating function used in MoND, but without needing any arbitrary constant. On the other hand, the second EUP exhibits both linear and quadratic corrections, leading to predictions consistent with the simple interpolating function of MoND. Clearly, given that EUP physics is largely heuristic, one should keep any scenario open. Hence, we expect that further hints towards the definitive answer may be provided by future cosmological experiments.

It is worth noting that both MoND and QI are claimed to solve the galaxy rotation problem without dark matter [25], but the latter does it without arbitrary adjustment. Moreover, QI seems also to explain the observed cosmic acceleration without needing dark energy [18,24]. In spite of these wellunderstood results, some other issues deserve further attention. As discussed above, the explanation for the modified inertia depends on the peculiar spacetime geometry seen by an accelerating body and, in particular, on the appearance of two relativistic horizons, the cosmic and Rindler ones. Nevertheless, the physical meaning of the latter is quite debated and its real existence often questioned, being related to the idealized notion of eternally accelerating motion (see Refs. $[4,55]$ for a discussion on the topic). Then, one may wonder to what extent the above predictions are actually testable. In this regard, a clue to a solution has been provided in Refs. $[56,57]$, where it has been suggested that artificial event horizons might be created by using the metamaterials proposed by Pendry et al. [58] and Leonhardt [59]. The idea is to engineer a material which reflects radiation in such a way an event horizon-like structure is formed. In the same way, another challenging perspective is to derive the intrinsic property of inertial mass of bodies (rather than modifications of its value) starting from similar considerations. These issues will be investigated in more detail in future works.

Data Availability Statement This manuscript has no associated data or the data will not be deposited. [Authors' comment: Data sharing is not applicable to this article as no datasets were generated or analyzed during the current study.]

Open Access This article is licensed under a Creative Commons Attribution 4.0 International License, which permits use, sharing, adaptation, distribution and reproduction in any medium or format, as long as you give appropriate credit to the original author(s) and the source, provide a link to the Creative Commons licence, and indicate if changes were made. The images or other third party material in this article are included in the article's Creative Commons licence, unless indicated otherwise in a credit line to the material. If material is not included in the article's Creative Commons licence and your intended use is not permitted by statutory regulation or exceeds the permitted use, you will need to obtain permission directly from the copyright holder. To view a copy of this licence, visit http://creativecomm ons.org/licenses/by/4.0/.

Funded by $\mathrm{SCOAP}^{3}$.

\section{Appendix A: EUP from metric expansion}

The EUP, as well as its GUP counterpart, are usually derived in literature using modified commutation relations for position and momentum introduced either ad hoc or on the basis of plausibility arguments. Following Ref. [54], in this Appendix we provides technical details on how to infer the form of the EUP from a given background metric.

Let us consider the metric in a generic $(1+1)$-dimensional curved spacetime

$d s^{2}=g_{t t} d t^{2}-g_{x x} d x^{2}$.

From Ref. [54], it is known that the quantum mechanics in the background (A.1) obeys the commutator

$[x, p]=i \hbar g_{x x}^{-1 / 2}$,

which gives the following uncertainty relation

$$
\begin{aligned}
& \Delta x \Delta p \geq \frac{1}{2}|\langle[x, p]\rangle| \\
& \Longrightarrow \Delta x \Delta p \geq \frac{\hbar}{2}\left|\left\langle g_{x x}^{-1 / 2}\right\rangle\right| .
\end{aligned}
$$

Clearly, in Minkowski flat spacetime, i.e., for $g_{x x}=1$, the ordinary quantum mechanics is recovered.

As a specific example, let us consider a modified uncertainty relation of the form

$$
\Delta x \Delta p \geq \frac{\hbar}{2}\left|1-\alpha \frac{\langle x\rangle}{l_{H}}+\frac{3 \alpha^{2}}{2} \frac{\left\langle x^{2}\right\rangle}{l_{H}^{2}}\right|,
$$

where $\alpha$ is a (dimensionless) positive constant and $l_{H}$ is a characteristic length-scale which emerges in the background under consideration. Note that the same relation has been proposed in Refs. [54,60] with only the absolute value of the linear term. Moreover, a specular Generalized Uncertainty Principle with $\left\langle p^{n}\right\rangle$ instead of $\left\langle x^{n}\right\rangle(n=1,2)$ is extensively used in literature (see Refs. [61,62] and therein), consistently with the predictions of string theory, black holes physics and doubly special relativity [63].

Now, by using Eq. (A.4), one can prove that Eq. (A.5) is consistent with the metric element

$g_{x x}=1+\frac{\alpha^{\prime} x}{l_{H}}$,

where $\alpha^{\prime}$ is a positive constant. Indeed, by taking into account the metric expansion up to the second order, the uncertainty relation (A.5) with $\alpha=\alpha^{\prime} / 2$ is recovered.

Equation (A.5) can now be recast in the form

$$
\begin{aligned}
\Delta x \Delta p \geq & \frac{\hbar}{2} \mid 1-\frac{\alpha}{l_{H}} \sqrt{\left\langle x^{2}\right\rangle-(\Delta x)^{2}} \\
& +\frac{3 \alpha^{2}}{2 l_{H}^{2}}\left(\langle x\rangle^{2}+(\Delta x)^{2}\right) \mid
\end{aligned}
$$




$$
\begin{aligned}
\gtrsim & \frac{\hbar}{2} \mid 1+\left(\frac{\alpha}{2 l_{H} \sqrt{\left\langle x^{2}\right\rangle}}+\frac{3 \alpha^{2}}{2 l_{H}^{2}}\right)(\Delta x)^{2} \\
& +\frac{3 \alpha^{2}}{2 l_{H}^{2}}\langle x\rangle^{2}-\frac{\alpha}{l_{H}} \sqrt{\left\langle x^{2}\right\rangle} \mid,
\end{aligned}
$$

where we have used the relation $(\Delta x)^{2}=\left\langle x^{2}\right\rangle-\langle x\rangle^{2}$. By making the approximation $\langle x\rangle \approx 0$, the above relation can be further manipulated, leading to

$$
\Delta x \Delta p \geq \frac{\hbar}{2}\left[1-\frac{\alpha}{2} \frac{\Delta x}{l_{H}}+\frac{3 \alpha^{2}}{2}\left(\frac{\Delta x}{l_{H}}\right)^{2}\right],
$$

where we have exploited the fact that the corrective terms are expected to be much smaller than unity. Clearly, for $\alpha=$ 0 and/or $\Delta x / l_{H} \ll 1$, the standard Heisenberg relation is recovered, as it should be.

On the other hand, one can show that the most common form of the EUP with only a quadratic term

$\Delta x \Delta p \geq \frac{\hbar}{2}\left[1+\frac{\gamma}{2}\left(\frac{\Delta x}{l_{H}}\right)^{2}\right]$,

is consistent with the topological structure of (anti)-de Sitter metric $[12,14]$. In this context, we remark that the additional term has a well-defined physical meaning, since it can be attributed to the cosmological constant $\Lambda \sim-1 / l_{H}^{2}$ and, thus, to the accelerating expansion of the Universe.

\section{References}

1. S.W. Hawking, Commun. Math. Phys. 43, 199 (1975)

2. W. Unruh, Phys. Rev. D 14, 870 (1976)

3. U. Leonhardt, Ann. Phys. 530, 1700114 (2018)

4. N.B. Narozhnyi, A.M. Fedotov, B.M. Karnakov, V.D. Mur, V.A. Belinsky, Phys. Rev. D 65, 025004 (2002)

5. D. Amati, M. Ciafaloni, G. Veneziano, Phys. Lett. B 197, 81 (1987)

6. D.J. Gross, P.F. Mende, Nucl. Phys. B 303, 407 (1988)

7. K. Konishi, G. Paffuti, P. Provero, Phys. Lett. B 234, 276 (1990)

8. M. Maggiore, Phys. Lett. B 319, 83 (1993)

9. A. Kempf, G. Mangano, R.B. Mann, Phys. Rev. D 52, 1108 (1995)

10. F. Scardigli, Phys. Lett. B 452, 39 (1999)

11. S. Capozziello, G. Lambiase, G. Scarpetta, Int. J. Theor. Phys. 39, 15 (2000)

12. B. Bolen, M. Cavaglia, Gen. Rel. Grav. 37, 1255 (2005)

13. Mi Park, Phys. Lett. B 659, 698 (2008)

14. S. Mignemi, Mod. Phys. Lett. A 25, 1697 (2010)

15. W.G. Unruh, Phys. Rev. Lett. 46, 1351 (1981)

16. C. Barcelo, S. Liberati, M. Visser, Living Rev. Rel. 8, 12 (2005)

17. A. Iorio, G. Lambiase, Phys. Lett. B 716, 334 (2012)

18. M. McCulloch, Mon. Not. Roy. Astron. Soc. 376, 338 (2007)
19. M. McCulloch, EPL 101, 59001 (2013)

20. J. Giné, M.E. McCulloch, Mod. Phys. Lett. A 31, 1650107 (2016)

21. M. McCulloch, EPL 115, 69001 (2016)

22. M. McCulloch, J. Giné, Adv. Stud. Theor. Phys. 14, 1 (2020)

23. M. Renda, Mon. Not. Roy. Astron. Soc. 489, 881 (2019)

24. M.E. McCulloch, EPL 90, 29001 (2010)

25. M.E. McCulloch, Astrophys. Space Sci. 342, 575 (2012)

26. M. Milgrom, Astrophys. J. 270, 365 (1983)

27. M. Milgrom, Astrophys. J. 270, 371 (1983)

28. J. Marino, A. Noto, R. Passante, Phys. Rev. Lett. 113, 020403 (2014)

29. M. Blasone, G. Lambiase, G.G. Luciano, Phys. Rev. D 96, 025023 (2017)

30. M. Blasone, G. Lambiase, G.G. Luciano, J. Phys: Conf. Ser. 956, 012021 (2018)

31. M. Blasone, G. Lambiase, G.G. Luciano, L. Petruzziello, Phys. Rev. D 97, 105008 (2018)

32. M. Blasone, G. Lambiase, G.G. Luciano, L. Petruzziello, Eur. Phys. J. C 80, 130 (2020)

33. J. Giné, EPL 121, 10001 (2018)

34. D. Chemisana, J. Giné, J. Madrid, EPL 130, 60002 (2020)

35. G. Dvali, C. Gomez, Fortsch. Phys. 61, 742 (2013)

36. G. Dvali, C. Gomez, Eur. Phys. J. C 74, 2752 (2014)

37. B. Famaey, J. Binney, Mon. Not. Roy. Astron. Soc. 363, 603 (2005)

38. G. Gentile, B. Famaey, W. de Blok, Astron. Astrophys. 527, A76 (2011)

39. R.J. Adler, D.I. Santiago, Mod. Phys. Lett. A 14, 1371 (1999)

40. F. Scardigli, R. Casadio, Class. Quant. Grav. 20, 3915 (2003)

41. S. Das, E.C. Vagenas, Phys. Rev. Lett. 101, 221301 (2008)

42. F. Scardigli, M. Blasone, G. Luciano, R. Casadio, Eur. Phys. J. C 78, $728(2018)$

43. G.G. Luciano, L. Petruzziello, Eur. Phys. J. C 79, 283 (2019)

44. V. Todorinov, P. Bosso, S. Das, Ann. Phys. 405, 92 (2019)

45. L. Buoninfante, G.G. Luciano, L. Petruzziello, Eur. Phys. J. C 79, 663 (2019)

46. M. Blasone, G. Lambiase, G.G. Luciano, L. Petruzziello, F. Scardigli, Int. J. Mod. Phys. D 29, 2050011 (2020)

47. D. Chemisana, J. Giné, J. Madrid, Int. J. Mod. Phys. D 29, 2050059 (2020)

48. L. Buoninfante, G. Lambiase, G.G. Luciano, L. Petruzziello, Eur. Phys. J. C 80, 853 (2020)

49. T. Zhu, J.R. Ren, M.F. Li, Phys. Lett. B 674, 204 (2009)

50. J. Mureika, Phys. Lett. B 789, 88 (2019)

51. M.P. Dabrowski, F. Wagner, Eur. Phys. J. C 79, 716 (2019)

52. W.S. Chung, H. Hassanabadi, Phys. Lett. B 793, 451 (2019)

53. B. Hamil, M. Merad, T. Birkandan, Phys. Scripta 95, 075309 (2020)

54. R.N. Costa Filho, J.P. Braga, J.H. Lira, J.S. Andrade, Phys. Lett. B 755, 367 (2016)

55. E.T. Akhmedov, D. Singleton, Pisma. Zh. Eksp. Teor. Fiz. 86, 702 (2007)

56. M.E. McCulloch, J. Br. Interplanet. Soc. 61, 373 (2008)

57. I.I. Smolyaninov, Opt. Lett. 44, 2224 (2019)

58. J.B. Pendry, D. Schurig, D.R. Smith, Science 312, 1780 (2006)

59. U. Leonhardt, Science 312, 1777 (2006)

60. W.S. Chung, Int. J. Theor. Phys. 58, 2575 (2019)

61. A.F. Ali, S. Das, E.C. Vagenas, Phys. Lett. B 678, 497 (2009)

62. S. Das, E.C. Vagenas, A.F. Ali, Phys. Lett. B 690, 407 (2010)

63. G. Amelino-Camelia, Living Rev. Rel. 16, 5 (2013) 